\title{
Proposição de um modelo metodológico de análise longitudinal de capacidade tecnológica e internacionalização
}

\author{
Rafael Kuramoto Gonzalez \\ Aluno de Doutorado da Fundação Getúlio Vargas - RJ, Pesquisador do IDRC, Professor-tutor do INEPAD e FGV \\ Online \\ rafael.gonzalez@fgv.br
}

\section{RESUMO}

Este artigo buscou realizar uma proposta metodológica de análise longitudinal de capacidades tecnológicas e internacionalização para empresas do setor de café solúvel situadas em economias emergentes. Para o desenvolvimento deste modelo, foram construídos dois instrumentos para análise dos dados. Para o estudo da internacionalização, foi utilizado o Modelo de Internacionalização de Empresas Produtoras Exportadoras Brasileiras construído pelo estudo de Kraus (2006). Para entender a trajetória de capacidade tecnológica, foi utilizado o Modelo de Capacidades Tecnológicas de Empresas de Economias Emergentes construído por Figueiredo (2002). Para a sua construção, foram necessárias uma extensa pesquisa com dados triangulados pela teoria, entrevistas, observação in loco e documentos técnicos. A partir do estudo realizado é possível determinar algumas sugestões de estudos empíricos sobre o processo de acúmulo de capacidade tecnológica e internacionalização para o enriquecimento de entendimento empírico.

Palavras-chave: Capacidade tecnológica, Internacionalização, Análise longitudinal.

\begin{abstract}
This article attempts to build a methodological proposal for longitudinal analysis of technological capabilities and internationalization of soluble coffee industry located in emerging economies. To develop this model, two instruments were built for data analysis. For the study of internationalization, was used the Internationalization Model of Brazilian exporting producer companies built by the study of Kraus (2006). To understand the trajectory of technological capability, it was used the Technological Capabilities Model in Emerging Economies firms built by Figueiredo (2002). For its development, was performed extensive research with triangulated data by theory, interviews, on-site observation and technical documents. From this study it is possible to determine some suggestions for empirical studies about the process of accumulation of technological capability and internationalization for the enrichment of empirical understanding.
\end{abstract}

Keywords: Technological capability, Internationalization, Longitudinal analysis.

\section{Introdução}

Uma perspectiva evolucionista e uma investigação sobre a acumulação de capacidades tecnológicas podem explicar a assimetria entre as empresas em termos de tecnologia e desempenho (DOSI, 1988; LALL 1992). Assim como há uma mudança das preocupações estatísticas sobre escolha, aquisição e uso de tecnologias para preocupações dinâmicas no decorrer do tempo sobre acumulação de capacidades e atividades inovadoras crescentes (BELL, 2006), há também um interesse substancial e crescente na pesquisa de evidências do fortalecimento de capacidades em economias em desenvolvimento (MARCELLE, 2005).
Vale ressaltar que as pesquisas sobre capacidades tecnológicas se dividem em duas grandes abordagens (DUTRÉNIT, 2000). Uma primeira tradição tem como foco de análise as organizações que operam em economias avançadas. Nesta literatura, as empresas-alvo de estudo são altamente inovadoras, formam a fronteira tecnológica internacional, e a literatura busca acrescentar explicações de como essas organizações podem tornar suas capacidades inovativas mais aprofundadas e ampliadas (Ex: PRAHALAD; HAMEL, 1990; LEONARD-BARTON, 1995; TEECE; PISANO; CHUEN, 1997; ZOLLO; WINTER, 2002). A segunda abordagem 
é constituída de estudos que focam empresas de economias emergentes. Esta literatura se difere da abordagem anterior, uma vez que as empresas que são o foco de análise ainda necessitam criar capacidades tecnológicas iniciais, começando pela acumulação de capacidades de produção, para somente depois, engajarem esforços para realizarem atividades inovadoras. Os estudos que procuram examinar as trajetórias de acumulação de capacidades tecnológicas e o papel das estratégias de aprendizagem neste processo avançaram de forma considerável nos últimos 15 anos. (Ex: HOBDAY, 1995; ARIFFIN; BELL, 1999; KIM, 1997, 1998; DUTRÉNIT, 2000; ARIFFIN, 2000; FIGUEIREDO, 2003, 2010; ARIFFIN; FIGUEIREDO, 2004; MARCELLE, 2005; CONSONI; QUADROS, 2006; TSEKOURAS, 2006; ROUSSEVA, 2008; DANTAS; BELL, 2009, 2011; QUADROS; CONSONI, 2009; YORUK, 2011) Porém, mesmo com a grande quantidade de pesquisas sobre o tema, estes estudos avançaram muito em descrevê-la, apresentando evidências empíricas das diferentes trajetórias realizadas, contudo, a literatura ainda apresenta limitações para dar explicações sobre a dinâmica e natureza dessas trajetórias tecnológicas, quais são os fatores influentes nesse processo, e qual a importância relativa de cada um dos fatores para determinar a acumulação de capacidades tecnológicas nas empresas inseridas em contexto de economias emergentes (BELL, 2006; BELL; FIGUEIREDO, 2012).

No campo da internacionalização, a dinâmica de pesquisas que buscam entender os motivadores, as barreiras, e o seu processo é tão amplo quanto o de capacidade tecnológica (JOHANSON; WIEDERSHEIM-PAUL, 1975; HYMER, 1976; JOHANSON; VAHLNE, 1977; VERNON, 1979; RUGMAN, 1980; BUCKLEY; CASSON, 1988; WELCH; LUOSTARINEN, 1988; ANDERSEN, 1993; LEERSNYDER, 1996; ANDERSSON, 2000; DUNNING, 2001; KOGUT, 2002; MCDOUGAL; OVIATT, 2005; KRAUS, 2006). Estes estudos buscaram entender a forma como empresas realizaram a busca, acesso, concretização e ampliação de sua internacionalização.

Desta forma, o objetivo geral do artigo é propor um modelo metodológico de Análise Longitudinal de Capacidade Tecnológica e Internacionalização para empresas do setor de café solúvel em economias emergentes. Os objetivos específicos são: (a) Propor um Modelo metodológico de Análise de capacidade tecnológica para o setor em questão e (b) Propor um Modelo metodológico de Análise de internacionalização para empresas de economia emergente. Acredita-se que uma investigação aprofundada do comportamento da empresa por estas duas perspectivas pode contribuir significativamente para o entendimento da co-evolução das estratégias de internacionalização e de capacitação tecnológica, apontando possíveis fatores influentes e inter-relações.
Para a realização dos objetivos propostos pelo artigo, o trabalho está dividido em quatro partes. Na segunda seção, é apresentada a revisão teórica sobre internacionalização. No terceiro bloco é apresentada a revisão teórica sobre capacidade tecnológica. Na quarta parte são apresentados o Modelo metodológico de análise de internacionalização e de Capacidade Tecnológica e na quinta seção as considerações finais.

\section{Internacionalização}

A Literatura sobre internacionalização é bastante ampla e diversificada. São várias as considerações feitas para elucidar o que é e como se dá o processo de internacionalização. Num enfoque evolucionista, a internacionalização pode ser considerada como um processo gradual de aquisição, integração e uso do conhecimento sobre operações e mercados internacionais, que incrementalmente aumenta o compromisso e o envolvimento com os mercados internacionais (JOHANSON; VAHLNE, 1977). Essas definições de internacionalização estão próximas do conceito dado por Lall (1980) onde o autor argumenta que o nível de internacionalização depende da combinação de vantagens de monopólio com as formas de implicações (em mercados estrangeiros). Kraus (2006, p. 60) entende a internacionalização como "um processo ocorrido ao longo do tempo, no qual a empresa produtora exportadora amplia o seu envolvimento e comprometimento em operações internacionais".

De acordo com Leonidou, Katsikeas e Samiee (2002), para qualquer organização obter sucesso em ambientes exportadores não é uma tarefa fácil, devido principalmente à natureza idiossincrática dos ambientes internacionais. Com mercados globalizados e as rápidas mudanças nas economias e nos sistemas políticos obrigaram novos estudos e definições no sentido de posicionamento geográfico, vantagens competitivas e a transferência de conhecimentos entre as economias (KOGUT, 2002). Essas empresas quando se tornam exportadoras, seus dirigentes e sua capacidade organizacional são desafiadas, tanto no conhecimento, experiência, intuição e sensibilidade para responder as mudanças cada vez mais rápidas dos mercados de acordo com Rocha e Christensen (1988; 2002).

Para este trabalho, foi considerada a conceitualização dada por Welch e Luostarinen (1988) onde a internacionalização pode ser entendida como o processo de envolvimento em operações internacionais e envolve atividades de comércio exterior, fluxos de capital, transferência de tecnologia, fluxo de informações e dados, alianças, fusões, aquisições, (IDE) Investimento Direto no Estrangeiro entre outros.

As teorias agrupadas na abordagem econômica de acordo com Dib e Carneiro (2006) se baseiam em critérios 
econômicos, onde prevalece o pensamento racional, ou pseudo-racional, para tratar da estratégia de internacionalização, com uma abordagem que é norteada pela busca da maximização de retornos financeiros e econômicos. Estes autores apontam três principais correntes: Teoria do Poder de Mercado (HYMER, 1976), Teoria da Internalização (BUCKLEY; CASSON, 1988; RUGMAN, 1980) e Teoria Eclética (DUNNING, 2001).

As teorias da Abordagem comportamental originaram-se, de acordo com Dib e Carneiro (2006), dos modelos de estágio ou modelos evolutivos. O mais citado e dentre eles é o modelo desenvolvido pela Escola de Uppsala (OVIATT; MCDOUGALL, 1999) por Johanson e Wiedersheim-Paul (1975) e Johanson e Vahlne (1977). Outras cinco contribuições teóricas com relevância no campo: Empreendedorismo Internacional de Mcdougall e Oviatt (2005), Teoria de Redes de Johanson e Vahlne (1977; 1990), I-model de Andersen (1993), Teorias de Grau de Envolvimento e Controle de Leersnyder (1996) e o Modelo de Internacionalização de empresas produtoras exportadoras brasileiras de Kraus (2006) que será utilizado para este trabalho. A discussão acerca dos méritos, limitações e contribuições das diferentes vertentes da literatura sobre internacionalização fogem do escopo deste trabalho. Para uma compreensão mais aprofundada, a literatura internacional oferece diferentes interpretações (ANDERSEN, 1997; FORSGREN, 2002).

O Modelo de Internacionalização de Empresas Produtoras Exportadoras Brasileiras foi construído pelo estudo de Kraus (2006), que montou uma abordagem a partir dos estudos da literatura nacional e internacional com a intenção de adequar à realidade brasileira. $\mathrm{O}$ autor considera que um modelo com poder explicativo para representar a realidade brasileira encontra respaldo na evidência de que no Brasil a grande maioria das empresas internacionaliza-se mediante o desenvolvimento de exportações (KRAUS, 2006, p. 34).

Kraus (2006) comenta em seu trabalho que em cada etapa existem estágios que podemos considerar como sub-etapas. O autor explica da seguinte forma cada etapa:

- Etapa do Pré-envolvimento - A organização está focada totalmente com o mercado local que considera seguro e conhecido. Esta etapa é composta por: Estágio Não-Exportadora e pelo Estágio Pré-Exportadora.

- Etapa do Envolvimento Passivo - Nesta etapa a empresa está envolvida com suas primeiras atividades de exportação, já tem uma estrutura, contatos e expôs seus produtos em estágios ou feiras. Esta etapa é composta por: Estágio Exportadora Irregular e Estágio Exportadora Passiva.
- Etapa do Envolvimento Ativo - Nesta etapa a empresa toma consciência de sua passividade e muda seu foco da produção para o mercado. Esta etapa é composta por: Estágio Exportadora Pré-Ativa e Estágio Exportadora Ativa.

- Etapa do Envolvimento Comprometido - Nesta etapa a empresa atua em vários mercados e busca adequar-se aos gostos e hábitos de seus consumidores, adaptando e desenvolvendo produtos e serviços de pós-venda específicos. Também é nesta fase que a empresa descobre oportunidades com a implantação de escritórios e unidades de produção.

\section{Capacidade tecnológica}

Enquanto as organizações da fronteira tecnológica já desenvolveram suas capacidades tecnológicas inovadoras, as empresas em processo de catching up ou em mercados de recente industrialização precisam se empenhar num processo gradativo de acumulação de capacidades tecnológicas para aproximar-se das fronteiras tecnológicas. Neste raciocínio, Bell, Larsson e Westphal (1984) apontaram que a organização em processo de industrialização, que no início opera suas atividades de forma pouca competitiva mundialmente (também conhecida como "infância industrial"), a acumulação de capacidades tecnológicas é ponto chave para alcançar a competitividade no mercado mundial (ou a "maturidade industrial"). E a aceleração da velocidade de acumulação de capacidade tecnológica é o meio pelo qual as organizações conseguem realizar o processo de catching up tecnológico e se aproximar ou alcançar a fronteira tecnológica (FIGUEIREDO, 2000).

Outra definição para capacidade tecnológica é encontrada na década de 70 com Katz (1976) que considera a capacidade tecnológica uma "atividade inventiva" ou um esforço criativo sistemático necessário para as melhorias da capacidade produtiva da empresa ou para obter novos conhecimentos em nível de produção. Já Dahlman e Westphal (1982) formularam o conceito de "domínio tecnológico", concretizado através do "esforço tecnológico" para assimilar, adaptar e/ou criar novas tecnologias, que podemos considerar uma definição análoga à "capacidade tecnológica" de Bell (1982) e Scott-Kemmis (1988) que consideram que a capacidade tecnológica inclui as aptidões e os conhecimentos incorporados nos trabalhadores, nas instalações e nos sistemas organizacionais, visando produzir mudanças tanto na produção, quanto nas técnicas utilizadas. Numa tentativa posterior, com a intenção de aprimorar o conceito de capacidade tecnológica, Westphal, Kim e Dahlman (1984, p. 5) define capacidade tecnológica como a "aptidão para usar efetivamente o conhecimento tecnológico". 
Seguindo nessa linha de pensamento da natureza intrínseca da tecnologia, Lall (1992) considera que a capacidade tecnológica de uma empresa é de natureza intrínseca, ou seja, requer um esforço deliberado interno à organização, e ela difere de uma para a outra, e por isso o conhecimento tecnológico não é completamente partilhado, transferido ou imitado entre as empresas. E esse processo de transferência envolve, obrigatoriamente, a aprendizagem endógena dos conhecimentos tecnológicos, que a princípio, em grande parte, não estão claramente definidos e disponíveis. Esse conhecimento tecnológico tem caráter estratégico e é dificilmente partilhado, transferido ou imitado entre as organizações. Lall (1982; 1987) considera a capacidade tecnológica um "esforço tecnológico interno" para dominar novas tecnologias, adaptando-as às condições locais, aperfeiçoando-as e até mesmo exportando-as. Essa conceituação dá a noção que a capacidade tecnológica é um conjunto de conhecimentos, aptidões e experiências tecnológicas acumuladas por meio de esforços tecnológicos endógenos, que podem ser conduzidos de forma explícita ou deliberada (COSTA, 2003).

Para este artigo, consideramos como mais apropriada a definição dada por Bell e Pavitt (1993) aonde é considerado capacidade tecnológica como os recursos necessários para gerar e gerenciar a mudança tecnológica, incluindo habilidades, conhecimentos e experiências, estruturas institucionais e as redes de ligações. Os recursos necessários para gerir esta mudança tecnológica estão alocados nos indivíduos e sistemas organizacionais (BELL; PAVITT, 1993; 1995). Bell e Pavitt (1993) diferenciam a "capacidade de produção" e a "capacidade inovadora", considerando capacidade de produção como as capacidades de rotina, que são as habilidades para as atividades operacionais e de produção de bens e serviços com determinado nível de eficiência, usando um aglomerado de dimensões: habilidades, equipamentos, especificações de produtos e de produção, sistemas e métodos organizacionais. No outro espectro, a capacidade inovadora refere-se a incorporação de recursos adicionais e distintos para gerar e gerenciar a mudança tecnológica.

$\mathrm{Na}$ literatura internacional também há um enorme esforço para a construção de teorias para entender o processo de capacitação tecnológica (ex. LALL, 1992; BELL; PAVITT, 1993; HOBDAY, 1995; ARIFFIN, 2000; MARCELLE, 2005), mas para este trabalho será utilizado o modelo construído por Figueiredo. De acordo com Figueiredo (2003) os estudos sobre capacidade tecnológica ganharam uma abordagem mais ampla na década de 90, o que levou à perspectiva de quatro dimensões de capacidade tecnológica: Sistema Físico, Sistema Organizacional, Mentes dos Indivíduos e Produtos e Serviços.
Depois de identificadas as dimensões da capacidade tecnológica, Figueiredo (2003) construiu um modelo que permite identificar e medir a capacidade tecnológica com base em atividades que a empresa é capaz de realizar ao longo de sua existência. O modelo distingue dois tipos de capacidade: Capacidades Rotineiras que compreende a habilidade de usar ou operar determinada tecnologia e Capacidade Inovadora que é a capacidade de adaptar ou desenvolver novos processos de produção, sistemas organizacionais, produtos, equipamentos e projetos de engenharia, ou seja, gerar e gerir a inovação tecnológica.

De acordo com Figueiredo (2003), o modelo é explicado da seguinte forma: As colunas representam as capacidades tecnológicas por função e as linhas os níveis de dificuldade. Essas categorias são medidas pelas atividades que expressam os níveis de capacidades ou, em outras palavras, o tipo de atividade que a empresa é capaz de realizar por sim mesma em diferentes intervalos de tempo. O modelo divide-se em sete níveis de capacidade para cinco funções tecnológicas.

Em Figueiredo (2004; 2005) é discutido o mérito dessa abordagem não convencional de análise da capacidade tecnológica comparado aos indicadores convencionais de análise e mensuração de P\&D e patentes. São apontados a irrelevância de indicadores com atividades de P\&D, patentes internacionais, laboratórios de P\&D formalmente estruturados e a abordagem estática de análise. Esse tipo de análise da relevância dos indicadores tem recebido grande atenção por parte de outros autores (Ex: LAESTADIUS, 1998; HIRSCH-KREINSEN; HAHN; JACOBSON, 2008; entre outros)

É ressaltado que o modelo não pressupõe uma seqüência de evolução linear ou que as capacidades sejam construídas ao mesmo tempo e na mesma velocidade para as diferentes funções tecnológicas (FIGUEIREDO, 2004).

\section{Modelo de análise longitudinal de capacidade tecnológica e internacionalização}

Para o desenvolvimento deste modelo, foram construídos dois instrumentos para análise dos dados. Para o estudo da internacionalização, foi utilizado o Modelo de Internacionalização de Empresas Produtoras Exportadoras Brasileiras do estudo de Kraus (2006). Para a operacionalização da análise dos dados, foi construído um Modelo de Caracterização das Etapas de Internacionalização (Quadro 1). Neste quadro são apresentados os critérios necessários para que a empresa possa ser categorizada de acordo com os níveis de internacionalização. 


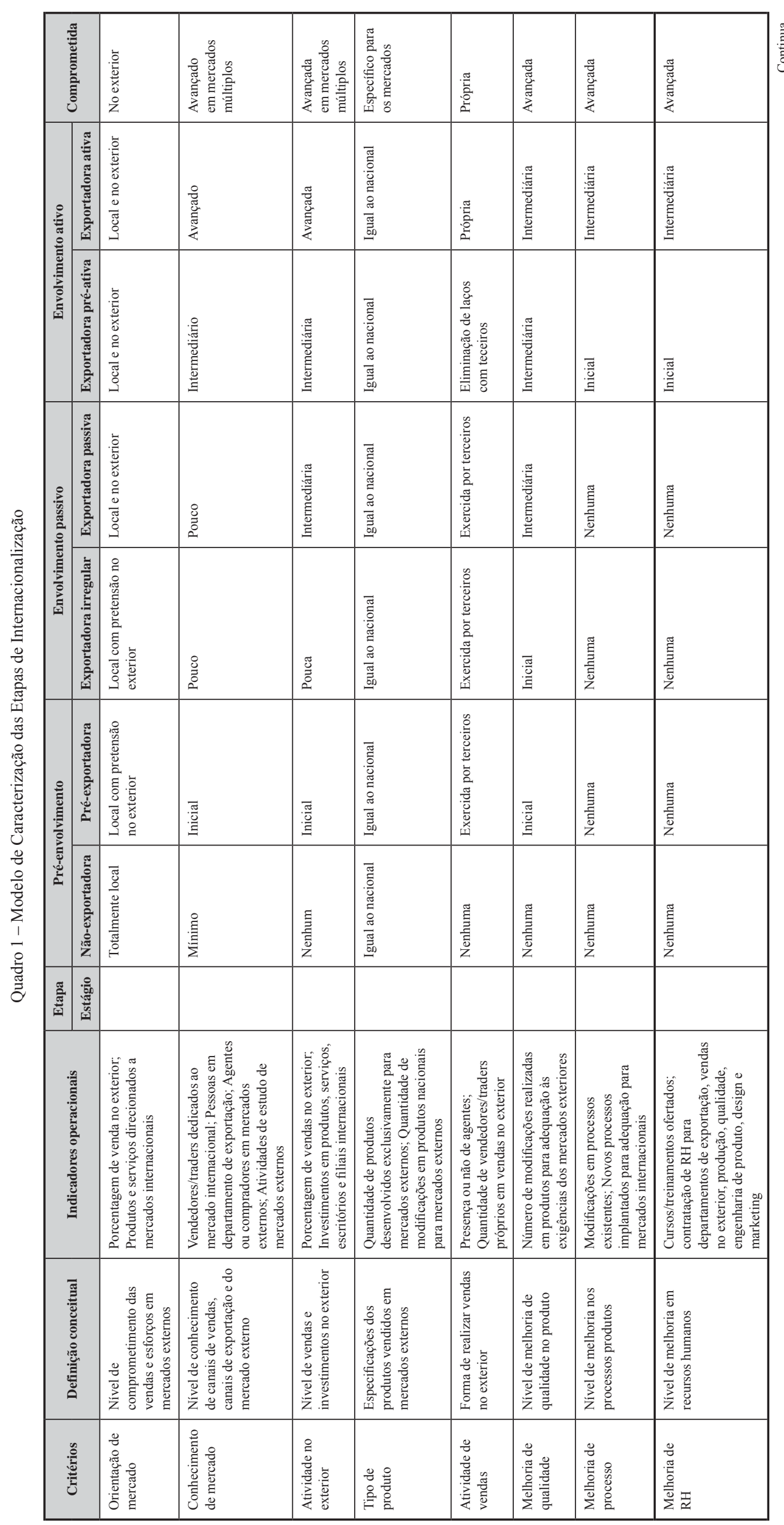


Produto \& Produção, vol. 14, n. 3, p. 07-18, out. 2013

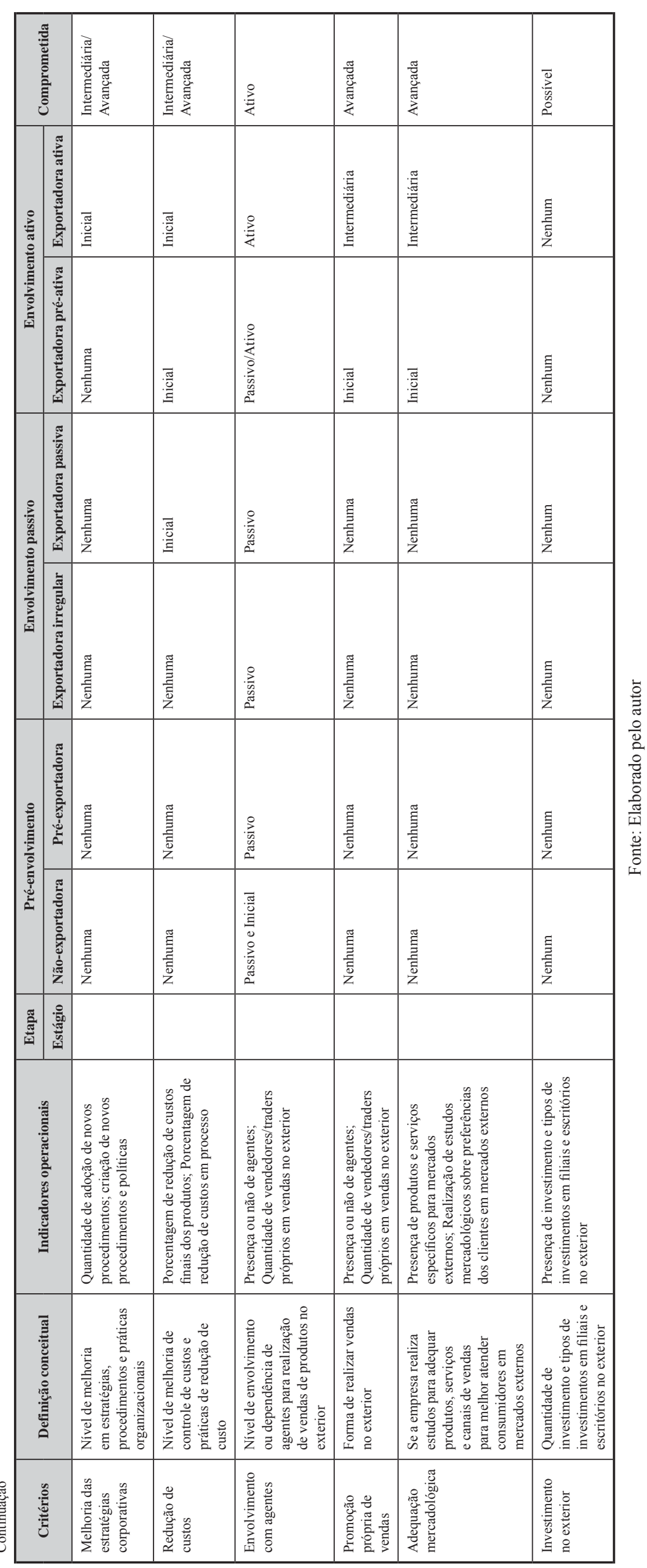


Para entender a trajetória de capacidade tecnológica da empresa, foi considerado que o Modelo de Capacidades Tecnológicas em Empresas de Economias Emergentes construído por Figueiredo (2003) era o mais apropriado. Para operacionalizar a análise dos dados, foi necessária realizar uma contextualização do modelo original construído pelo autor para a indústria siderúrgica para a indústria estudada. Desta forma, foi construído o Modelo Descritivo de Capacidade Tecnológica de Empresas do Setor de Café Solúvel (Quadro 2), que é

Quadro 2 - Modelo Descritivo de Capacidade Tecnológica

\begin{tabular}{|c|c|c|c|c|c|c|}
\hline \multirow{3}{*}{$\begin{array}{l}\text { Níveis de Capacidade } \\
\text { Tecnológica }\end{array}$} & \multicolumn{6}{|c|}{ Função Tecnológica } \\
\hline & \multirow[b]{2}{*}{\begin{tabular}{l}
\multicolumn{1}{c}{ Produto } \\
Produção de Café \\
Solúvel em Pó \\
com especificações \\
amplamente aceitas \\
e com possível \\
fornecimento para \\
mercados internacionais \\
preparado pelo processo \\
Spray Drying.
\end{tabular}} & \multirow{2}{*}{\begin{tabular}{l}
\multicolumn{1}{c}{ Processo } \\
Coordenação de Rotina \\
na Fábrica e Absorção da \\
Capacidade Produtiva da \\
Fábrica.
\end{tabular}} & \multirow{2}{*}{\begin{tabular}{l}
\multicolumn{1}{c}{ Equipamentos } \\
Instalação de \\
Equipamentos para \\
Produção Básica de \\
Café Solúvel Spray e \\
Liofilizado.
\end{tabular}} & \multirow[b]{2}{*}{\begin{tabular}{l}
\multicolumn{1}{c}{ Investimentos } \\
Decisão sobre localização \\
da fábrica e dos \\
escritórios, realizando \\
estudos de viabilidade \\
técnico-econômica. \\
Decisão de compra de \\
Maquinário de Qualidade \\
Mundial.
\end{tabular}} & \multicolumn{2}{|l|}{ P\&D } \\
\hline & & & & & $\begin{array}{l}\text { Capacidade de instalação de } \\
\text { tecnologias e absorção de } \\
\text { transferência de tecnologias. } \\
\text { Desenvolvimento de } \\
\text { projetos em conjunto. } \\
\text { Necessidade de assistência } \\
\text { técnica terceirizada. } \\
\text { Melhoria de tecnologias } \\
\text { instaladas. }\end{array}$ & 范. \\
\hline (2) Renovado & $\begin{array}{l}\text { Produção de Café } \\
\text { Solúvel Liofilizado com } \\
\text { especificações simples e } \\
\text { com fornecimento para } \\
\text { mercados internacionais } \\
\text { preparado pelo processo } \\
\text { Freeze Drying. }\end{array}$ & $\begin{array}{l}\text { Coordenação } \\
\text { Aprimorada da Fábrica. } \\
\text { Implantação inicial de } \\
\text { ferramentas de qualidade } \\
\text { básicas. Uso pioneiro de } \\
\text { Sistemas Informatizados } \\
\text { e de Sistemas de } \\
\text { Integração. }\end{array}$ & $\begin{array}{l}\text { Instalação de } \\
\text { Equipamentos para } \\
\text { Produção Aprimorada } \\
\text { de Café Solúvel Spray } \\
\text { e Extrato. Perfuração } \\
\text { de Poço Artesiano para } \\
\text { atendimento da Fábrica. }\end{array}$ & $\begin{array}{l}\text { Decisão sobre } \\
\text { participação de Capital } \\
\text { Estrangeiro, criando } \\
\text { sinergias para ampliação } \\
\text { produtiva e comercial. }\end{array}$ & $\begin{array}{l}\text { Primeiras iniciativas de } \\
\text { P\&D próprias. Capacidade } \\
\text { de criação e implantação } \\
\text { de novas tecnologias. } \\
\text { Concepção de órgão } \\
\text { especializado em novas } \\
\text { tecnologias e prestação de } \\
\text { assistência técnica. }\end{array}$ & 5 \\
\hline (3) Extrabásico & $\begin{array}{l}\text { Produção de diversos } \\
\text { tipos de Café Solúvel } \\
\text { com especificações } \\
\text { moderadas e com ampla } \\
\text { aceitação em mercados } \\
\text { internacionais com a } \\
\text { Marca. }\end{array}$ & $\begin{array}{l}\text { Pequenas Adaptações } \\
\text { e Intermitentes em } \\
\text { Processos Produtivos da } \\
\text { Fábrica. Consolidação } \\
\text { da implantação de } \\
\text { ferramentas de qualidade } \\
\text { básicas e implantação } \\
\text { de novas técnicas } \\
\text { organizacionais como } \\
\text { o JIT, Kanban, TQM e } \\
\text { outros. }\end{array}$ & $\begin{array}{l}\text { Instalação de } \\
\text { Equipamento } \\
\text { para Produção } \\
\text { Aprimorada de Café } \\
\text { Solúvel Liofilizado. } \\
\text { Manutenção Técnica } \\
\text { de equipamentos } \\
\text { autônoma. }\end{array}$ & $\begin{array}{l}\text { Decisão sobre } \\
\text { investimentos em } \\
\text { melhorias do ativo } \\
\text { existente e na criação de } \\
\text { novos ativos, ampliando } \\
\text { capacidade produtiva, } \\
\text { sistema de produção e } \\
\text { portfólio de produtos. }\end{array}$ & $\begin{array}{l}\text { Formalização de } \\
\text { Departamento de P\&D } \\
\text { própria. Concepção } \\
\text { de projetos em Célula } \\
\text { de Produção Piloto, } \\
\text { Laboratório Químico e } \\
\text { Laboratório de Design. }\end{array}$ & \\
\hline (4) Pré-intermediário & $\begin{array}{l}\text { Produção de Óleo } \\
\text { e Extrato de Café } \\
\text { com especificações } \\
\text { complexas e com } \\
\text { aceitação em mercados } \\
\text { internacionais preparado } \\
\text { pelo processo de } \\
\text { Crioconcentração. }\end{array}$ & $\begin{array}{l}\text { Ampliação sistemática } \\
\text { da capacidade produtiva. } \\
\text { Uso de ferramentas para } \\
\text { promover a Segurança } \\
\text { no Trabalho e de } \\
\text { Preservação do Meio } \\
\text { Ambiente. }\end{array}$ & $\begin{array}{l}\text { Instalação de } \\
\text { Equipamentos para } \\
\text { Produção Complexa } \\
\text { de Café Solúvel Spray } \\
\text { e Óleo. Realização } \\
\text { de Manutenção } \\
\text { Preventiva. Perfuração } \\
\text { de Poço artesiano de } \\
\text { grande profundidade. }\end{array}$ & $\begin{array}{l}\text { Decisão de investimentos } \\
\text { em Empresas horizontais } \\
\text { e verticais do Setor, } \\
\text { ampliando mercado } \\
\text { de atuação horizontal } \\
\text { e cobertura de setores } \\
\text { correlatos de importância } \\
\text { estratégica. }\end{array}$ & $\begin{array}{l}\text { Formalização de } \\
\text { Departamento de Pesquisa } \\
\text { Aplicada capacitada para } \\
\text { entregar estudos, projetos, } \\
\text { produtos, processos e } \\
\text { soluções inovadoras } \\
\text { referência em seu Segmento } \\
\text { Industrial Nacionalmente. }\end{array}$ & \\
\hline (5) Intermediário & $\begin{array}{l}\text { Produção de Café } \\
\text { Solúvel Aglomerado } \\
\text { com especificações } \\
\text { muito rígidas e } \\
\text { penetração em vários } \\
\text { mercados internacionais } \\
\text { preparado pelo processo } \\
\text { de concentração a frio do } \\
\text { extrato de café líquido. }\end{array}$ & $\begin{array}{l}\text { Aprimoramento } \\
\text { contínuo de processo. } \\
\text { Desenho de Sistemas } \\
\text { automatizados estáticos. } \\
\text { Implantação de Sistemas } \\
\text { de Integração em toda } \\
\text { a fábrica. Obtenção de } \\
\text { Certificações ISO. }\end{array}$ & $\begin{array}{l}\text { Instalação de } \\
\text { Equipamentos para } \\
\text { Produção Complexa } \\
\text { de Café Aglomerado e } \\
\text { para Produção de Nível } \\
\text { Mundial em sua cadeia } \\
\text { produtiva. }\end{array}$ & $\begin{array}{l}\text { Decisão de âmbito } \\
\text { internacional com } \\
\text { a instalação de } \\
\text { escritórios em mercados } \\
\text { internacionais chave. }\end{array}$ & $\begin{array}{l}\text { Formalização de } \\
\text { Departamento de Pesquisa } \\
\text { Aplicada capacitada para } \\
\text { entregar estudos, projetos, } \\
\text { produtos, processos e } \\
\text { soluções inovadoras } \\
\text { referência em todos } \\
\text { os Setores Produtivos } \\
\text { Nacionalmente. }\end{array}$ & 豙 \\
\hline $\begin{array}{l}\text { (6) Intermediário } \\
\text { Superior }\end{array}$ & $\begin{array}{l}\text { Produção de diversos } \\
\text { tipos de Café Solúvel } \\
\text { com especificações } \\
\text { de alto padrão e } \\
\text { desenvolvidas de acordo } \\
\text { com as especificações } \\
\text { solicitadas pelo cliente } \\
\text { internacional. Obtenção } \\
\text { de novos produtos } \\
\text { através de P\&D. }\end{array}$ & $\begin{array}{l}\text { Consolidação do } \\
\text { Sistema operacional } \\
\text { total. Engajamento em } \\
\text { melhorias contínuas } \\
\text { inovadoras em } \\
\text { processos através } \\
\text { de P\&D. Obtenção } \\
\text { de Certificações de } \\
\text { Mercados Internacionais. }\end{array}$ & $\begin{array}{l}\text { Instalação de } \\
\text { Equipamentos para } \\
\text { Produção de Nível } \\
\text { Mundial de Café } \\
\text { Solúvel Liofilizado e } \\
\text { de Eficiência Ecológica } \\
\text { Superior. }\end{array}$ & $\begin{array}{l}\text { Decisão de âmbito } \\
\text { internacional com a } \\
\text { instalação de unidades } \\
\text { produtoras em mercados } \\
\text { internacionais chave. }\end{array}$ & $\begin{array}{l}\text { Formalização de } \\
\text { Departamento de Pesquisa } \\
\text { Aplicada capacitada } \\
\text { para entregar estudos, } \\
\text { projetos, produtos, } \\
\text { processos e soluções } \\
\text { inovadoras referência em } \\
\text { seu Segmento Industrial } \\
\text { Internacionalmente. }\end{array}$ & \\
\hline (7) Avançado & $\begin{array}{l}\text { Produção de diversos } \\
\text { tipos de Café Solúvel } \\
\text { com especificações de } \\
\text { Nível e Reconhecimento } \\
\text { Mundial. Obtenção de } \\
\text { novos produtos através } \\
\text { de P\&D de classe } \\
\text { mundial. }\end{array}$ & $\begin{array}{l}\text { Sistema de Produção } \\
\text { de classe mundial. } \\
\text { Referência em desenho } \\
\text { e desenvolvimento } \\
\text { de novos processos } \\
\text { baseados em P\&D de } \\
\text { classe mundial. }\end{array}$ & $\begin{array}{l}\text { Instalação de } \\
\text { Parque Fabril de } \\
\text { Nível Mundial. } \\
\text { Desenvolvimento de } \\
\text { projetos de P\&D para } \\
\text { novos equipamentos e } \\
\text { componentes. Prestação } \\
\text { de assistência técnica } \\
\text { para outras empresas. }\end{array}$ & $\begin{array}{l}\text { Decisão de transferência } \\
\text { de capital e produção } \\
\text { para mercados globais, } \\
\text { procurando explorar } \\
\text { vantagens competitivas } \\
\text { de países e regiões. }\end{array}$ & $\begin{array}{l}\text { Formalização de } \\
\text { Departamento de Pesquisa } \\
\text { Aplicada capacitada para } \\
\text { entregar estudos, projetos, } \\
\text { produtos, processos e } \\
\text { soluções inovadoras } \\
\text { referência em todos } \\
\text { os Setores Produtivos } \\
\text { Internacionalmente. }\end{array}$ & \\
\hline
\end{tabular}


dividido em conjuntos de capacidade, que são as capacidades de rotina e as capacidades inovadoras. A divisão entre capacidade rotineira e capacidade inovadora é explicada a seguir e seus critérios seguem a orientação teórica de Bell e Pavitt (1993; 1995).

A construção do modelo descritivo de capacidades tecnológicas foi realizada após imersão no campo empírico do setor industrial, buscando a compreensão da dinâmica tecnológica e gerencial, além do apoio incondicional de especialistas da área para validação do modelo. Esse grupo de especialistas (cada um com o mínimo de 15 anos de experiência na área) contou com a ajuda de 2 (dois) ex-presidentes, 4 (quatro) ex-diretores, 1 (um) diretor industrial, 1 (um) gerente de suprimentos, manutenção e logística, 1 (um) coordenador de qualidade e processos, 1 (um) coordenador de P\&D, 1 (um) coordenador de treinamento e desenvolvimento, 1 (um) assessor do sindicato de produtores de café, 1 (um) assessor da associação de produtores de café solúvel, 4 (quatro) professores-pesquisadores doutores especialistas da área.

Para a função PRODUTO, as capacidades inovativas iniciam a partir do $6^{\circ}$ Nível. Com a "commoditização" dos demais produtos de café solúvel, somente empresas capazes de realizar pesquisas para especificar seus produtos de acordo com o interesse de cada cliente internacional podem ser consideradas realmente inovadoras.

Para a função PROCESSO, as capacidades inovativas iniciam a partir do $5^{\circ}$ Nível. Empresas inovadoras buscam obter certificações de qualidade e certificações internacionais para garantir à clientes internacionais a procedência de seus produtos e poderem se destacar no mercado internacional. As demais ferramentas e ações de qualidade são somente o apoio para que a empresa possa criar esse diferencial competitivo.

Para a função EQUIPAMENTO, as capacidades inovativas iniciam a partir do $5^{\circ}$ Nível. Considera-se empresas inovativas nesta função quando elas tem um parque fabril equipado com maquinário que possibilite a produção de todas as variações de café solúvel encontradas no mercado nacional e internacional e várias seções do seu processo produtivo que estejam equipadas com máquinas de Nível Mundial.

Para a função INVESTIMENTO, as capacidades inovativas iniciam a partir do $4^{\circ}$ Nível. Empresas que tenham capacidade de tomar decisões de expansão de seus negócios de forma horizontal e vertical com relação ao seu setor podem ser consideradas inovadoras. Essas empresas buscam abrir sua capacidade de visão estratégica, focando em ações de cobertura de mercado, expansão de participação via setores correlatos, fortalecimento da marca e ações para estreitar suas relações com clientes.

Para a função $P \& D$, as capacidades inovativas iniciam a partir do $3^{\circ}$ Nível. São consideradas empresas inovativas quando elas alocam recursos e esforços para possuir um Departamento de P\&D formalizado.

\section{Considerações finais}

Este artigo buscou realizar uma proposta metodológica de análise longitudinal de capacidades tecnológicas e internacionalização para empresas específicas do setor de café solúvel situadas em economias emergentes. Foram utilizadas várias referências bibliográficas para a definição das teorias utilizadas, e a construção dos modelos e de sua metodologia. Podemos ressaltar que nem todos os trabalhos estudados serviram de fonte direta para explicar o fenômeno da internacionalização e capacidade tecnológica, mas ajudaram a elucidar como esses dois processos poderiam estar interligados e verificar as lacunas e transposição dos dois assuntos.

Para a construção dos modelos de análise metodológica, foram necessárias uma extensa pesquisa com dados triangulados pela teoria, entrevistas, observação in loco e documentos técnicos. A conclusão destes modelos, assim como a sua validação só foram possíveis através das valiosas contribuições de especialistas do setor de café solúvel, assim como de pesquisadoresprofessores interessados tanto no tema de inovação tecnológica, mudança técnica e internacionalização. Cabe ressaltar que o modelo descritivo foi construído considerando os aspectos e particularidades exclusivamente do setor de Café Solúvel, sendo considerados aspectos relevantes para esse setor da indústria, não podendo ser generalizados para outros setores sem a devida validação.

Para este artigo foram buscados dois objetivos para que a pesquisa atingisse ao Objetivo Geral proposto: (a) Propor um Modelo metodológico de Análise de capacidade tecnológica para o setor em questão e (b) Propor um Modelo metodológico de Análise de internacionalização para empresas de economia emergente.

Para o primeiro objetivo, com as evidências empíricas encontradas e apoiados nas teorias neo-schumpterianas de capacidades tecnológicas, e do modelo construído por Figueiredo, foi possível construir um modelo com o objetivo de traçar a trajetória de acumulação de capacidade tecnológica de empresas do setor de café solúvel em cinco funções tecnológicas diferentes: Produto, Processo, Equipamentos, Investimentos e P\&D.

Para o segundo objetivo, com os dados coletados na pesquisa e apoiados nas teorias de internacionalização, 
do modelo construído por Kraus e com a construção do modelo descritivo, foi possível construir um modelo com a sensibilidade necessária para captar as sutilezas do processo de internacionalização de empresas de economias emergentes.

É enfatizado que a forma como foram construídos e determinados os Modelos Metodológicos foram totalmente baseados para o estudo de empresas de café solúvel de economias emergentes. Erros, limitações e interpretações são de total responsabilidade do autor.

A partir do estudo realizado é possível determinar algumas sugestões de possibilidades de pesquisa sobre o processo de acúmulo de capacidade tecnológica e internacionalização: (I) Desenvolvimento de estudos em empresas brasileiras do setor. (II) Desenvolvimento de estudos em empresas de outros países do mundo de economias emergentes; (III) Desenvolvimento de modelos com base quantitativa para aplicação em larga escala; (IV) Estudo de aprofundamento com outras perspectivas, interpretações e teorias para, uma vez que o trabalho realizado não engloba todas as considerações possíveis; (V) Desenvolvimento de modelos para áreas industriais correlatas; (VI) Desenvolvimento de um modelo que permita a análise de mais de um setor paralelamente, mas que respeite as especificidades de todos os setores e que tenha a menor distorção possível.

\section{Referências}

ANDERSEN, O. On the internationalization process of firms: A critical analysis. Journal of International Business Studies, v. 24, n. 2, p. 209-231, 1993.

ANDERSSON, S. The internationalization of the firm from an entrepreneurial perspective. International Studies of Management \& Organization, v. 30, n. 1, Spring, p. 63-92, 2000.

ARIFFIN, N. The Internationalisation of Innovative Capabilities: The Malaysian Electronics Industry. $\mathrm{PhD}$ Thesis - SPRU, University of Sussex, Brighton, 2000.

ARIFFIN, N.; BELL, M. Firms, Politics and Political Economy: Patterns of Subsidiary-Parent Linkages and Technological Capability-building in Electronics TNC Subsidiaries in Malaysia. In: JOMO, K. S.; FELKER, G.; RASIAH, R. (Eds.). Industrial Technology Development in Malaysia. UK: Routledge, p. 150-190, 1999.

ARIFFIN, N.; FIGUEIREDO, P. N. Internationalization of innovative capabilities: counter-evidence from the electronics industry in Malaysia and Brazil. Oxford Development Studies, v. 32, n. 4, p. 559-583, 2004.
BELL, M. Technical Change in Infant Industries: A Review of the Empirical Evidence. SPRU, University of Sussex, Mimeo, 1982.

BELL, M. Time and technological learning in industrialising countries: how long does it take? How fast is it moving (if at all)? International Journal of Technology Management, v. 36, n. 1-3, p. 25-39, 2006.

BELL, M.; FIGUEIREDO, P. N. Innovation Capability Building and the Role of Learning Processes in Latecomer Firms: Recent Empirical Contributions and implications for research. Canadian Journal of development Studies/Revue canadienne d'études du développement, v. 33, n. 1, p. 13-40, 2012.

BELL, M.; LARSSON, R.; WESTPHAL, L.E. Assessing the Performance of Infant Industries. World Bank Staff Working Papers n. 666. Washington: The World Bank, 1984.

BELL, M.; PAVITT, K. Technological accumulation and industrial growth: contrast between developed and developing countries. Industrial and Corporate Change, v. 2, n. 2, p. 157-210, 1993.

BELL, M.; PAVITT, K. The Development of Technological Capabilities. In: HAQUE, I. (Ed.). Trade, Technology and International Competitiveness. The World Bank: Washington DC, 1995.

BUCKLEY, P. J.; CASSON, M. The limits of explanation: testing the internalization theory of the multinational enterprise. Journal of International Business Studies, v. 19, n. 2, p. 181-193, 1988.

CONSONI, F.; QUADROS, R. Multinational R\&D activity and product development capabilities in Brazil: a case-study focused on carmakers. International Journal of Technology Management, v. 36, n. 1/2/3, 2006.

COSTA, I. Empresas multinacionais e capacitação tecnológica na indústria brasileira. 188 p. Tese (Pós-Graduação em Política Científica e Tecnológica) - Universidade Estadual de Campinas, Campinas, 2003.

DAHLMAN, C.; WESTPHAL, L. Technological effort in industrial development: an interpretative survey of recent research. In: STEWART, F.; JAMES, J. (Eds.). The economics of new technology in developing countries. London: Frances Pinter, p. 105-137, 1982.

DANTAS, E.; BELL, M. Latecomer firms and the emergence and development of knowledge networks: The case of Petrobras in Brazil. Research Policy, v. 38, p. $829-844,2009$. 
DANTAS, E.; BELL, M. The Co-Evolution of Firm-Centered Knowledge Networks and Capabilities in Late Industrializing Countries: The Case of Petrobras in the Offshore Oil Innovation System in Brazil. World Development, doi:10.1016/j.worlddev.2011;02;002, 2011.

DIB, L. A.; CARNEIRO, J. Avaliação Comparativa do Escopo Descritivo e Explanatório dos Principais Modelos de Internacionalização de Empresas. In: Anais do XXX ENANPAD. Salvador: ANPAD, 2006.

DOSI, G. The microeconomic sources and effects of innovation. An assessment of some recent findings. Brigthon: SPRU, University of Sussex, DRC Discussion Paper n. 33, 1985.

DOSI, G. The nature of the innovative process. In: DOSI, G.; FREEMAN, C.; NELSON, R.; SILVERBERG, G.; SOETE, L. (Eds.). Technical Change and Evolutionary Theory. London: Pinter Publishers, 1988.

DUNNING, J. H. The eclectic paradigm of international production: past, present, and future. International Journal of the Economics of Business, v. 8, n. 2, p. 173-190, 2001 .

DUTRÉNIT, G. Learning and knowledge management in the firm: from knowledge accumulation to strategic capabilities. Cheltenham, UK; Northampton, MA, USA: Edward Elgar, 2000.

FIGUEIREDO, P. N. Trajetórias de acumulação de competências tecnológicas e os processos subjacentes de aprendizagem: revisando estudos empíricos. Revista de Administração Pública, FGV, v. 34, n. 1, p. 7-33, jan./fev., 2000.

FIGUEIREDO, P. N. Aprendizagem Tecnológica e Inovação Industrial em Economias Emergentes: uma Breve Contribuição para o Desenho e Implementação de Estudos Empíricos e Estratégias no Brasil. Revista Brasileira de Inovação, v. 3, n. 2, p. 323-361, jul./dez., 2004.

FIGUEIREDO, P. N. Acumulação Tecnológica e Inovação Industrial: conceitos, mensuração e evidência no Brasil. São Paulo em Perspectiva, v. 19, n. 1, p. 54-69, 2005.

FIGUEIREDO, P. N. Does technological learning pay off? Inter-firm differences in technological capability-accumulation paths and operational performance improvement. Research Policy, Inglaterra, v. 31, n. 1, p. 73-94, 2002a.

FIGUEIREDO, P. N. Learning processes features and technological capability accumulation: explaining inter-firm differences. Technovation, Inglaterra, v. 22, n. 11, p. $685-698,2002$ b.

FIGUEIREDO, P. N. Aprendizagem Tecnológica e Performance Competitiva. Rio de Janeiro: Editora FGV, 2003.

FIGUEIREDO, P. N. What Recent Research Does and Doesn't Tell Us about Rates of Latecomer Firms Capability Accumulation. Asian Journal of Technology Innovation, Seoul, v. 15, p. 161-195, 2007.

FIGUEIREDO, P. N. Industrial Policy Changes and Firm-Level Technological Capability Development: Evidence from Northern Brazil. World Development, v. 36, p. 55-88, 2008.

FIGUEIREDO, P. N. Discontinuous innovation capability accumulation in latecomer natural resource-processing firms. Technological Forecasting and Social Change, v. 77, p. 1090-1108, 2010.

FIGUEIREDO, P.N. The Role of Dual Embeddedness in the Innovative Performance of MNE Subsidiaries: Evidence from Brazil. Journal of Management Studies, Oxford Print, v. 48, p. 417-440, 2011.

FORSGREN, M. The concept of learning in the Uppsala internationalization process model: a critical review. International Business Review, v. 11, n. 3, p. 267-277, 2002.

HIRSCH-KREINSEN, H.; HAHN, K.; JACOBSON, D. The low-tech issue. In: HIRSCH-KREINSEN, H.; JACOBSON, D. (Eds.). Innovation in Low-Tech firms and Industries. Cheltenham: Edward Elgar, 2008.

HOBDAY, M. Innovation in East Asia: the challenge to Japan. Aldershot: Edward Elgar, 1995.

HYMER, S. The international operations of national firms: a study of direct foreign investment. Doctorate Thesis, Cambridge, MA: The MIT Press, 1976.

JOHANSON. J.; VAHLNE, J. The internationalization process of the firm: a model of knowledge development and increasing market commitment. Journal of International Business Studies, v. 8, p. 23-32, Spring/Summer, 1977.

JOHANSON. J.; VAHLNE, J. The Mechanism of Internationalization. International Marketing Review, v. 7, n. 4, p. 11-24, 1990.

JOHANSON, J.; WIEDERSHEIM-PAUL, F. The internationalization of the firm: Four Swedish Cases. Journal of Management Studies, v. 12, n. 3, p. 305-322, 1975. 
KATZ, J. Importación de tecnología, aprendizaje y industrialización dependiente. México: Fondo de Cultura Económica, 1976.

KIM, L. The dynamics of Samsung's technological learning in semiconductors. California Management Review, v. 39, n. 3, p. 86-100, 1997.

KIM, L. Crisis construction and organizational learning: capability building in catching-up at Hyundai Motor. Organization Science, New York, v. 9, n. 4, p. 506-521, jul./ago., 1998.

KOGUT, B. International International Management and Strategy. In: PETTIGREW, A.; WHITTINGTON, R. (Eds.). Strategy and Management. London: Sage Publications, p. 261-278, 2002.

KRAUS, P. G. O Processo de Internacionalização das Empresas: o caso brasileiro. Revista de Negócios, Blumenau, v. 11, n. 2, p. 25-47, abr./jun., 2006.

LALL, S. Learning to industrialise: the acquisition of technological capability by India. London: Macmillan, 1987.

LALL, S. Monopolistic Advantages and Involvement by U.S. Manufacturing Industry. Oxford Economic Papers (New Series), v. 32, n. 1, 1980.

LALL, S. Technological learning in the Third World: some implications of technology exports. In: STEWART, F.; JAMES, J. (Eds.). The economics of new technology in developing countries. London: Frances Printer, 1982.

LALL, S. Technological Capabilities and Industrialization. World Development, v. 20, n. 2, p. 165- 186, 1992.

LAESTADIUS, S. The relevance of science and technology indicators: the case of pulp and paper. Research Policy, v. 27, p. 385-395, 1998.

LEERSNYDER, J. M. Marketing international. Paris: Dalloz, 1996.

LEONARD-BARTON, D. Wellsprings of Knowledge: Building and Sustaining the Sources of Innovation. Boston, MA: Harvard Business School Press, 1995.

LEONIDOU, L.C.; KATSKIEAS, C.S.; SAMIEE, S. Marketing strategy determinants of export performance: a meta-analysis. Journal of Business Research, v. 55, n. 1, p. 51-67, 2002.
MARCELLE, G. How do Telecom firms build capabilities? Lessons from Africa. Telecommunications Policy, v. 29, p. 549-572, 2005.

MCDOUGALL, P. P.; OVIATT, B. M. The internationalization of entrepreneurship. Journal of International Business Studies, v. 36, n. 1, p. 2-8, 2005.

NELSON, R.; WINTER, S. An Evolutionary Theory of Economic Change. Cambridge: Harvard University Press, 1982.

PRAHALAD, C.; HAMEL, G. The Core Competence of the Corporation. Harvard Business Review, v. 3, n. 90, p. 79-91, 1990.

QUADROS, R.; CONSONI, F. Innovation capabilities in the Brazilian automobile industry: a study of vehicle assemblers' technological strategies and policy recommendations. International Journal of Technological Learning, Innovation and Development, v. 2, n. 1-2, p. 53-75, 2009.

ROCHA, A.; CHRISTENSEN, C. Por que as empresas exportam. In: ROCHA, A. et al (Org.). Gerência de exportação no Brasil. Rio de Janeiro: UFRJ, 1988. p. 86-114.

ROCHA, A.; CHRISTENSEN, C. Como as empresas brasileiras exportam: a revisão dos estudos sobre exportação (1978-1990). In: ROCHA, A. (Org.). A internacionalização das empresas brasileiras: estudos de gestão internacional. Rio de Janeiro: Mauad, 2002. p. 85-118.

ROUSSEVA, R. Identifying technological capabilities with different degrees of coherence: The challenge to achieve high technological sophistication in latecomer software companies (based on the Bulgarian case). Technological Forecasting and Social Change, v. 75, p. 1007-1031, 2008

RUGMAN, A. M. A New Theory of the Multinational Enterprise: Internationalization Versus Internalization. Columbia Journal of World Business, v. 15, n. 1, p. 23, 1980 .

SCOTT-KEMMIS, D. Learning and the accumulation of technological capacity in Brazilian pulp and paper firms. World Employment Programme Research. Working Paper n. 187, p. 2-22, 1988.

TEECE, D.; PISANO, G.; SHUEN, A. Dynamic capabilities and strategic management. Strategic Management Journal, v. 18, n. 7, p. 509-533, 1997. 
TSEKOURAS, G. Gaining competitive advantage through knowledge integration in a European industrialising economy. International journal of technology management, v. 36, n. 1-3, p. 126-147, 2006.

VERNON, R. The product cycle hypothesis in a new international environment. Oxford Bulletin of ECOnomics and Statistics, v. 41, n. 4, p. 255-267, 1979.

WELCH, L. S.; LUOSTARINEN, R. Internationalization: Evolution of a Concept. Journal of General Management, v. 14, n. 2, p. 34, Winter 1988.

WESTPHAL, L.; KIM, L.; DAHLMAN, C. J. Reflections of Korea's acquisition of technological capability.
Washington, DC: World Bank Research Department, Economics and Research Staff (Report DRD77), 1984.

ZOLLO, M.; WINTER, S. Deliberate learning and the evolution of dynamic capabilities. Organization Science, v. 13, p. 339-351, 2002.

YORUK, E. E. The influence of technological capabilities on the knowledge network component of innovation systems: evidence from advanced materials in Turkey, Int. J. of technological Learning, Innovation and Development, v. 4, in press, 2011.

Recebido em: 13/01/2012. Aceito em: 04/06/2013. 